\title{
Campo de investigación en tecnologías de información y comunicación: estrategia de gobernanza en la Universidad de Medellín
}

\author{
Field research in information and communication technologies: \\ governance strategy at Universidad de Medellín
}

\author{
Liliana González P. ${ }^{1} \quad$ Sandra Arango $^{2} \quad$ Claudia Vásquez $^{2} \quad$ Jennifer Ospina $^{1}$ \\ Recibido 30 de octubre de 2013, aceptado 20 de junio de 2014 \\ Received: October 30, 2013 accepted: June 20, 2014
}

\begin{abstract}
RESUMEN
Como parte de su plan de desarrollo y del Proyecto Educativo Institucional, la Universidad de Medellín, Colombia, requiere orientar sus esfuerzos de investigación en Tecnologías de Información y Comunicación -TIC-. Actualmente no hay claridad sobre las fortalezas que deben ser potenciadas, lo cual dificulta la focalización de capacidades. Se hace necesario construir un nicho en el que la Institución sea reconocida y claramente diferenciada con respecto a otras entidades de educación superior. Se propone la estructuración del campo en TIC como estrategia para la gobernanza desde el punto de vista de investigación dentro de la Universidad. En este artículo se presenta un conjunto de tendencias de investigación como directriz institucional para enfocar la capacidad instalada en cuanto a recurso humano calificado, infraestructura y procesos. Para tal efecto se está usando una perspectiva cualitativa bajo enfoque metodológico hermenéutico. Hallazgos parciales revelan que se debe investigar en temáticas asociadas a educación, infraestructura tecnológica, regulación y políticas, medio ambiente, desarrollo de software, gestión y administración de las organizaciones y contenidos digitales. Cada temática se desagrega en un conjunto de sub-temáticas para mayor claridad. Con este mapeo de tendencias será posible direccionar los esfuerzos de la Universidad de Medellín y obtener reconocimiento por los avances que se alcancen en cuanto a investigación de vanguardia asociada a las TIC. Este direccionamiento está acompañado de un plan de comunicaciones para un despliegue adecuado en toda la comunidad logrando apropiación del tema en todos los niveles.
\end{abstract}

Palabras clave: Campo de investigación, tendencias de investigación, TIC, gobernanza, estrategia.

\begin{abstract}
As a part of its development plans and educational Project, Universidad de Medellín, Colombia, intends to focus its research efforts on information and communications technology-ICT-. There is still a lack of information about the strengths that need to be enhanced, which makes focalization of capabilities a difficult task. The University needs to create a niche in which it would be recognized and distinguished from other institutions. To achieve this aim, a strategy in order to guide the ICT research activities within the University is proposed. This article reflects on a number of research trends to be taken into account as organizational directives that allow an optimal use of resources and infrastructure. For this purpose a qualitative perspective and a hermeneutic methodological approach are used. Former research findings reveal that Universidad de Medellin should research on topics related to education, technology infrastructure, policy and regulatory environment, software development, digital content, management and administration of organizations. In order to show a clearer view, every topic is divided into subgroups. With this mapping of the trends it would be possible for the University to optimize its efforts on
\end{abstract}

\footnotetext{
1 Grupo Arkadius. Facultad de Ingeniería. Universidad de Medellín. Carrera 87 № 30-65. Medellín, Colombia. E-mail: ligonzalez@udem.edu.co; jenny918_1@ hotmail.com

2 Grupo E-Virtual. Facultad de Comunicaciones. Universidad de Medellín. Carrera 87 Nº 30-65. Medellín, Colombia. E-mail: sarango@udem.edu.co; cpvasquez@udem.edu.co
} 
IT research and to be recognized for its achievements in that field. This initiative should be completed by an effective communication strategy that informs the community on all the levels of the topics.

Keywords: Research field, research trends, ICT, governance, strategies.

\section{INTRODUCCIÓN}

El Estado colombiano reconoce que el acceso y uso de las Tecnologías de la Información y las Comunicaciones -TIC-, el despliegue y uso eficiente de la infraestructura, el desarrollo de contenidos y aplicaciones, la protección a los usuarios, la formación de talento humano en estas tecnologías y su carácter transversal, son pilares para la consolidación de las sociedades de la información [1].

Entendida la Universidad como una organización de conocimiento, es natural que la incorporación de TIC en su operación dinamice sus procesos. Pero más allá de pensar que estas tecnologías se ubican en el nivel de apoyo de la planeación estratégica, en la Universidad de Medellín las TIC son pensadas además como un factor diferenciador al que se debe dar relevancia desde el proceso misional de investigación.

Para un mayor aprovechamiento de las TIC en la Universidad de Medellín y como buena práctica de gobernanza de las mismas, se busca su incorporación y alineación desde el Proyecto Educativo Institucional -PEI- y el Plan de Desarrollo como principales cartas de navegación de la organización [2].

Buscando hacer realidad "la pertinencia a nivel investigativo" comprometida en una de las finalidades educativas del PEI, la institución está apostando a la estructuración de un campo de investigación en TIC, lo cual se constituye en una estrategia de gobernanza.

Este artículo esboza un conjunto de tendencias de investigación en TIC como directriz institucional para enfocar la capacidad instalada en cuanto a recurso humano calificado, infraestructura y procesos buscando construir un nicho en el que la Universidad de Medellín sea reconocida y claramente diferenciada con respecto a otras instituciones de educación superior.

Para tal efecto, en el primer apartado se presentan los referentes conceptuales que soportan la propuesta. La metodología y hallazgos parciales en la estructuración del campo de investigación en TIC se explican posteriormente. Por último se presentan las conclusiones.

\section{REFERENTES CONCEPTUALES}

En esta sección se presentan algunos conceptos que facilitan el entendimiento del esquema propuesto para la gobernanza de las TIC desde el punto de vista de investigación en la Universidad de Medellín. Las Tecnologías de Información y Comunicación -TIC- se definen como un conjunto de herramientas y procesos para acceder, recuperar, guardar, organizar, manipular, producir, intercambiar y presentar información por medios electrónicos. Esto incluye hardware, software y telecomunicaciones en la forma de computadores y programas tales como aplicaciones multimedia y sistemas de bases de datos [3].

Otra definición de TIC obedece a un conjunto de tecnologías capaces de producir, almacenar y transmitir información digital y, asimismo, de funcionar en red como plataforma organizativa de las diversas actividades humanas y sociales que soportan, en el espacio tiempo que ellas mismas generan [4].

Una universidad, como organización de conocimiento, debe contar con un esquema claro para la gestión de las TIC en orden a garantizar el funcionamiento óptimo de sus procesos de apoyo, y también aquellos catalogados como misionales, es decir, la docencia, la investigación, la extensión y la internacionalización.

Para un mayor aprovechamiento de las TIC en la Universidad de Medellín se ha incorporado el concepto de gobernanza, que según la Organización Internacional de Normalización (ISO) se define como el sistema que dirige y controla el uso actual y futuro de las Tecnologías de Información, para ponerlo al servicio de la organización y supervisar su alineación con los planes estratégicos [5]. Complementando lo anterior, COBIT agrega que 
la gobernanza TIC permite orientar las actividades y garantizar la alineación con los objetivos y el mandato de la organización; la habilitación de la organización accediendo a mejores oportunidades y maximizando beneficios, el uso responsable de los recursos TIC y la gestión adecuada de los riesgos relacionados con TIC.

Como parte de las estrategias de gobernanza se busca la incorporación y alineación de las TIC desde el Proyecto Educativo Institucional -PEI- y el Plan de Desarrollo como principales cartas de navegación de la organización [2].

El PEI es el elemento integrador de todos los procesos institucionales que posibilita la aproximación a las finalidades educativas propias, destinadas a satisfacer las necesidades personales y sociales. Es el instrumento que promueve una gestión educativa coherente y eficaz [6]. Está constituido por cuatro núcleos, tal como se muestra en la (Figura 1):

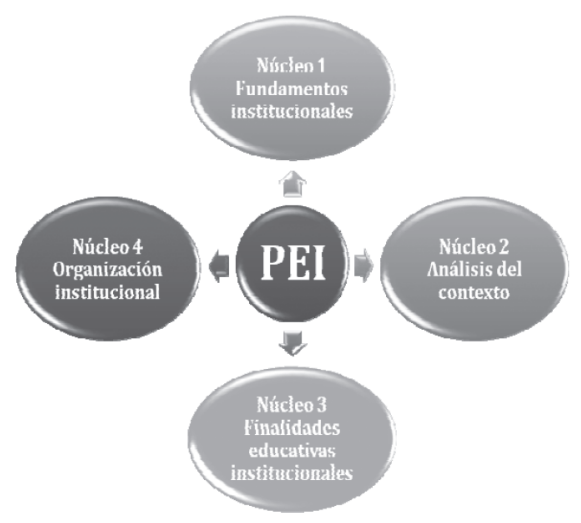

Figura 1. Composición del Proyecto Educativo Institucional -PEI- Universidad de Medellín.

El Plan de Desarrollo está conformado por un nivel estratégico y uno operativo buscando aportar al cumplimiento de las finalidades educativas (núcleo 3), como se evidencia en la Figura 2.

Por cada finalidad educativa presente en el PEI se configuran a nivel estratégico una serie de políticas, con objetivos bien definidos, que a su vez requieren estrategias y se materializan mediante proyectos.

Respecto a la fase operativa, todo adquiere mayor nivel de detalle mediante la definición de productos,

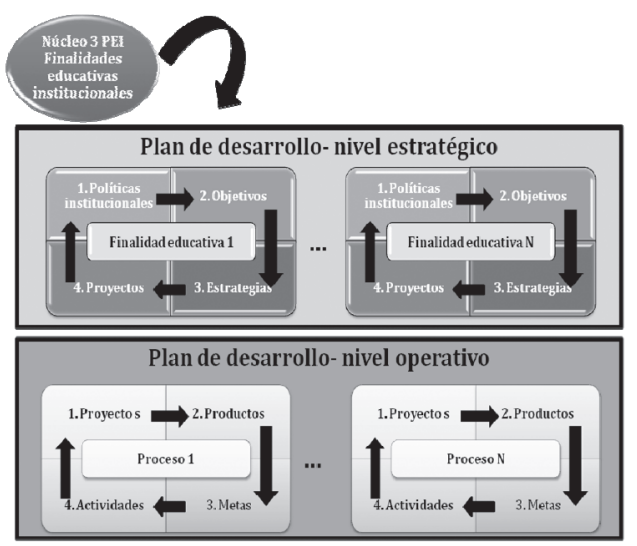

Figura 2. Estructura del plan de desarrollo Universidad de Medellín.

metas y actividades para lograr los proyectos planteados desde el aspecto estratégico.

Como finalidades relacionadas con la investigación se tienen: promover la investigación científica de alto nivel y pertinente, dirigida a fortalecer comunidades científicas vinculadas a la sociedad del conocimiento.

La composición a nivel estratégico para cubrir esta finalidad se enuncia a continuación:

\section{Políticas:}

$\checkmark$ Proyectar los resultados de la investigación a nivel nacional e internacional.

$\checkmark \quad$ La Investigación de la Universidad accederá a las nuevas fuentes del conocimiento y de la innovación.

$\checkmark$ La Universidad garantizará procesos investigativos de calidad.

\section{Objetivos:}

$\checkmark$ Lograr de la investigación en los diferentes programas, resultados que impacten en el medio.

$\checkmark \quad$ Integrar la investigación con el medio.

$\checkmark$ Asegurar la calidad de los proyectos de investigación.

$\checkmark$ Definir la estructura funcional de la investigación.

\section{Estrategias:}

$\checkmark$ Articular la investigación con el pregrado, postgrado y educación para el trabajo y el desarrollo humano.

$\checkmark$ Fortalecer la participación de la comunidad académica en el proceso de investigación. 
$\checkmark$ Garantizar un adecuado acompañamiento, vigilancia e intervención del proceso investigativo.

$\checkmark$ Evaluar y sistematizar el proceso de investigación.

$\checkmark$ Ejercer seguimiento y control a los procesos investigativos.

$\checkmark$ Diseñar propuestas académico-administrativas para garantizar el funcionamiento de la actividad investigativa.

Otra finalidad relacionada con investigación es: fomentar la interacción con la sociedad en los campos académico, investigativo y de proyección, en todos los ámbitos (nacional e internacional) y con responsabilidad social.

La investigación tiene la siguiente configuración estratégica:

\section{Políticas:}

$\checkmark$ La Universidad de Medellín desarrollará estrategias permanentes y específicas tendientes a lograr la internacionalización de la investigación a través de acciones y procesos investigativos y científicos de proyección internacional, así como de perspectiva internacional para el desarrollo local e institucional, movilidad, intercambios y migración de conocimiento

\section{Objetivos:}

$\checkmark$ Transferir y proyectar en la comunidad interna y externa los desarrollos científicos y tecnológicos.

$\checkmark$ Ofrecer productos y servicios de acuerdo con las necesidades del entorno.

$\checkmark$ Desarrollar la cultura de la planeación, ejecución, evaluación y control, en el desarrollo de sus funciones sustantivas.

$\checkmark$ Establecer y desarrollar procesos y acciones específicas para la interacción de la Universidad con el mundo en sus campos de acción e interés.

\section{Estrategias:}

$\checkmark$ Establecer vínculos interinstitucionales con comunidades científicas de desarrollo tecnológico y de cooperación internacional.

$\checkmark$ Desarrollar proyectos de investigación con impacto a las nuevas realidades del mundo moderno.

Al pensar en las TIC desde el punto de vista investigativo, es posible evidenciar que se trata de una de las áreas más promisorias a nivel nacional e internacional además de ser un componente potenciador y transversal de las demás áreas del conocimiento [7].

Al hablar del "campo de TIC" se hace referencia a un conjunto de ámbitos estratégicos de vanguardia relacionados con las TIC, que integran problemáticas de carácter inter y trans-disciplinario en los contextos: regional, nacional e internacional [8].

En las siguientes secciones se presenta el proceso realizado para detectar las tendencias de investigación en TIC y algunos hallazgos parciales interesantes.

\section{EL CAMPO TIC: UNA BUENA PRÁCTICA DE GOBERNANZA}

\section{Metodología}

En la investigación para detectar las tendencias en TIC se emplea una perspectiva cualitativa, conocida también como comprensiva. El enfoque metodológico utilizado es el hermenéutico. Según Gadamer, en la hermenéutica, "toda labor de conceptualización persigue, en principio, el consenso posible, el acuerdo posible e, incluso, debe basarse en un consenso si se ha de lograr que las personas se entiendan entre sî́" [9].

Partir de la hermenéutica para estructurar el campo de TIC en la Universidad de Medellín permite pensar en las TIC como el objeto de estudio que corresponde al "todo", y en las temáticas que la conforman como sus "partes". Es de anotar que durante la aplicación del círculo hermenéutico es posible ajustar cada vez más las temáticas que se propondrán para el campo de TIC de la Universidad de Medellín. Dicho ciclo está basado en los prejuicios, el análisis, la comparación, la reflexión, la comprensión, la interpretación y la síntesis (Figura 3) [10].

1. Prejuicios: compete a los saberes previos de los investigadores en el tema. Este proyecto cuenta con un grupo interdisciplinario de la institución compuesto por profesionales en áreas de la ingeniería y la educación. Esto permite abarcar de manera integral las temáticas del campo de TIC.

2. Análisis: en esta etapa se comienza el reconocimiento de algunas temáticas y sub- 


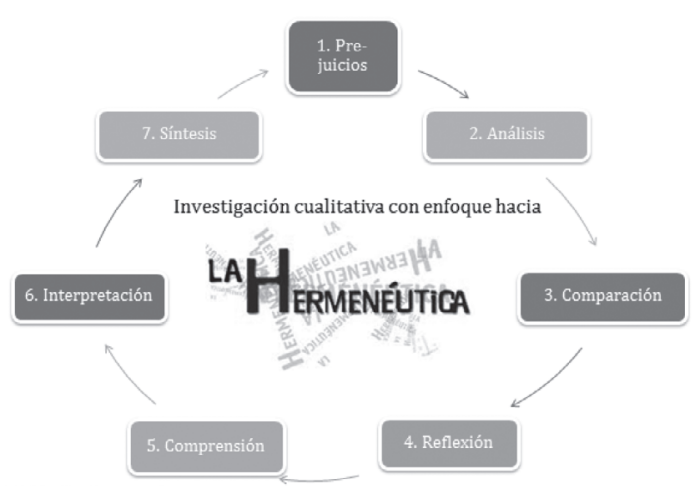

Figura 3. Círculo hermenéutico.

temáticas pertenecientes a las tecnologías de información y comunicación. Es posible que durante el proceso investigativo muchos elementos identificados como importantes sean filtrados, modificados, eliminados como parte de la dinámica normal en la consolidación de información.

3. Comparación: los conocimientos previos (prejuicios) de los investigadores, el estado actual de la investigación en la Universidad de Medellín y el rastreo documental permitirán continuar con la consolidación de las temáticas y sub-temáticas del campo TIC.

4. Reflexión: este proceso también conocido como autocrítica es el que permite incluir la voz del investigador después de los procesos de análisis y comparación, es la posibilidad de permear lo existente a través de comentarios propios basados en la experiencia que se va adquiriendo en el proceso de investigación.

5. Comprensión: en este punto se reconocen nuevas temáticas y sub-temáticas que constituyen el campo TIC, además de consolidar referentes teóricos y conceptuales necesarios en la investigación.

6. Interpretación: las TIC son un asunto muy amplio para investigar ya que incluyen numerosos temas y elementos, en esta etapa se utilizan todos los hallazgos obtenidos a partir de diferentes fuentes (libros, artículos, entrevistas, interacción con grupos de interés) para orientarlos al contexto de la UdeM.

7. Síntesis: es la etapa final, por lo tanto aquí se consolidará el campo TIC como producto principal de esta investigación. Durante la aplicación de la metodología se realizarán ajustes permanentes. Todo esto dependerá del análisis, comparación, reflexión e interpretación de nuevas fuentes documentales y resultados de los instrumentos de investigación.

La unidad de análisis (a la cual se le aplica el círculo hermenéutico ya descrito) está compuesta por las fuentes documentales que corresponden a artículos y proyectos sobre las TIC publicados a nivel local, regional, nacional e internacional.

Algunos referentes significativos que indican tendencias en TIC son:

- Antioquia Virtual Siglo XXI [11].

- Medellín Digital [12].

- Plan Estratégico Vive Digital [13].

- Horizon Report [7].

- Plan Estratégico de Electrónica, Telecomunicaciones e Informática Colciencias.

- Plan Estratégico de Ciencia, Tecnología e Innovación de Medellín 2011-2021 [14].

Hasta el momento de escritura de este artículo se ha realizado el rastreo a más de 200 documentos. En el enfoque cualitativo no se define un límite numérico para la revisión documental, por el contrario, es una etapa continua durante la investigación.

Otras fuentes fundamentales en la unidad de análisis son los grupos de investigación de la Universidad de Medellín, sus líneas y sus proyectos relacionados con el campo TIC; los investigadores internos de la Universidad con sus percepciones, imaginarios y aportes que le puedan hacer al campo TIC desde su área de conocimiento; expertos en el tema con los cuales se genere diálogo para indagar sobre las TIC a nivel social, gubernamental, económico, entre otros.

En cuanto a los instrumentos de investigación se cuenta con:

- Ficha de rastreo documental.

- Ficha resumen del rastreo/matriz de tendencias vs. referentes.

- Guión para entrevistas a expertos en el tema.

- Grupos focales con los investigadores de la UdeM. 
Para comprender mejor la metodología de la investigación a continuación se presenta un diagrama en notación BPMN (Figura 6).

Existen tres actividades paralelas (esto es, se pueden realizar en cualquier momento y no interfieren en el desarrollo de otras actividades) en el proyecto: hacer seguimiento y control, construir la memoria metodológica y generar los productos de divulgación. Dichos pasos están en permanente construcción y ejecución.

Tras indicar las actividades paralelas se especifican aquellas secuenciales, es decir, las que se ejecutan de forma ordenada, ya que son dependientes de otras. Se indican los instrumentos usados para abordar cada una, además de los productos obtenidos. El objetivo final es la construcción del campo TIC, y su respectiva transferencia a toda la comunidad.

\section{TENDENCIAS DE INVESTIGACIÓN EN TIC}

Conforme a la revisión documental junto con un análisis de las áreas de conocimiento y grupos de investigación presentes en la Universidad de Medellín, los hallazgos parciales muestran que la institución debe enfocarse en siete temáticas asociadas a las TIC (Figura 4).

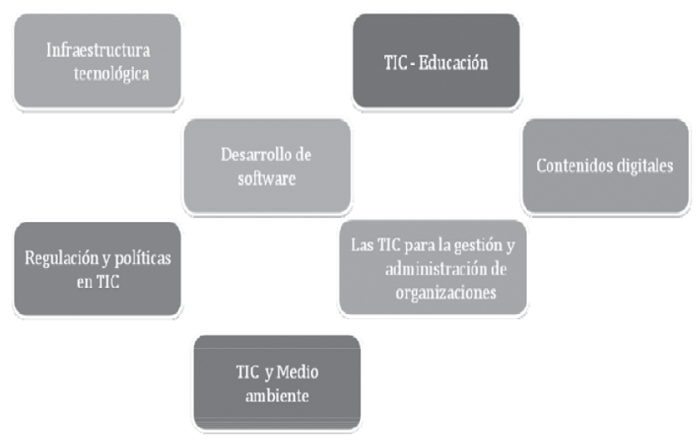

Figura 4. Temáticas de investigación en TIC.

\section{- Infraestructura tecnológica}

Este término hace referencia a un conjunto de elementos de hardware, software, telecomunicaciones y servicios que en conjunto dan soporte a las aplicaciones (sistemas informáticos) de una organización, buscando una configuración adecuada para recolectar, manipular, almacenar y procesar datos que se convertirán en información y permitirán la operación y la toma de decisiones en el nivel estratégico (Figura 5).

\section{- Desarrollo de software}

Puesto que cualquier solución en TIC es una dupla de hardware y software, en esta temática del campo se propone investigar para la identificación de requisitos de información en la organización, modelado conceptual de datos y su integración con elementos estructurales como bases de datos y demás elementos de una solución software. También se incluyen tópicos asociados a la administración de las fuentes de información mediante herramientas de apoyo.

Todo lo anterior debe estar conectado con la lógica del negocio para que la información en realidad se constituya en un activo para la organización. De otro lado, las soluciones ofrecidas deben construirse sobre estándares y mejores prácticas promulgadas por la ingeniería de software (Figura 7).

\section{- Regulación y políticas en el uso de las TIC}

Si bien en Colombia hay leyes de derechos de autor, no son suficientes frente al amplio espectro de productos y artefactos derivados de la creación, apropiación y uso de las TIC, por lo que se hace indispensable tener una regulación que proteja los contenidos digitales, además de trabajar en pro de la uniformidad de las licencias de propiedad intelectual por parte de las entidades de gestión colectiva. También se hace necesario impulsar una armonización de las leyes de protección de datos y mejorar la seguridad en el entorno digital. Como parte de este tópico hay que evitar delitos informáticos y tener normas claras para combatir dichos delitos con herramientas legales y de la informática forense [1]. Otro punto interesante es la creación de códigos de conducta para el uso de los sitios web, sin perjudicar el desarrollo de las TIC pero protegiendo la dignidad de las personas. En este último tema entra la protección a los menores de edad, especialmente en lo relacionado con la pornografía infantil (Figura 8) [15].

\section{- TIC-Educación}

En esta temática surge la necesidad de investigar para la generación de una cultura de uso y apropiación de las TIC, buscando que la comunidad aprenda a utilizar servicios antes no disponibles, incorporándolos a su vida cotidiana, sea cual sea su estrato, actividad 


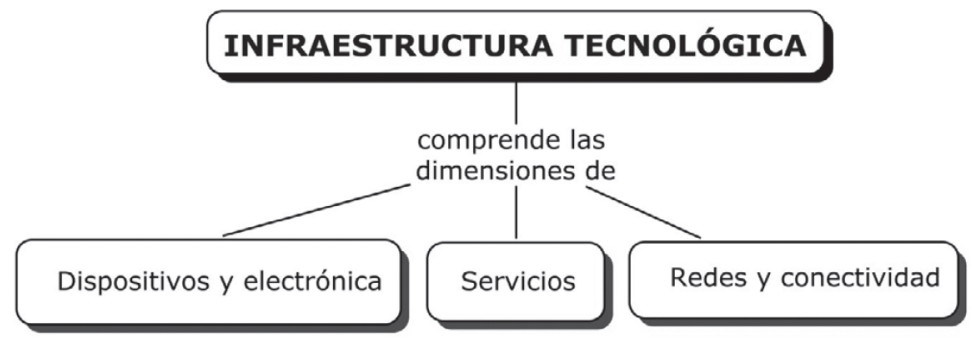

Figura 5. Mapa de la temática "Infraestructura tecnológica".

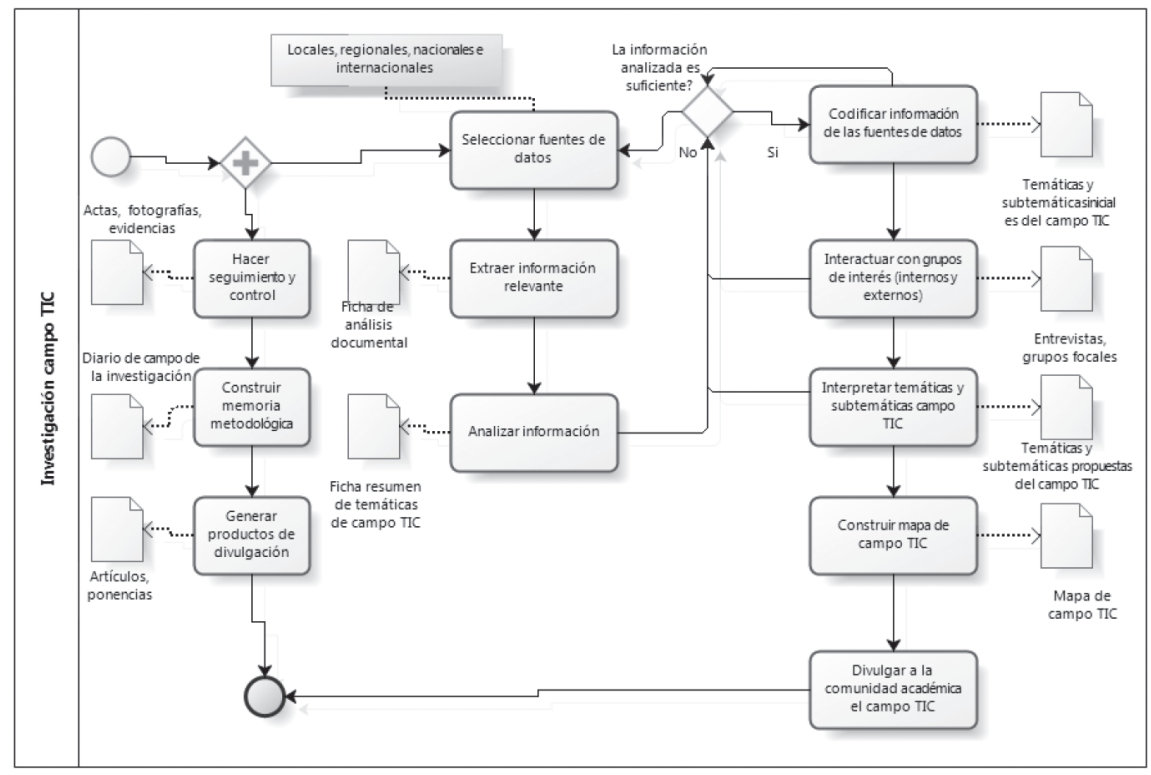

Figura 6. Diagrama de procesos asociado a la metodología de la investigación.

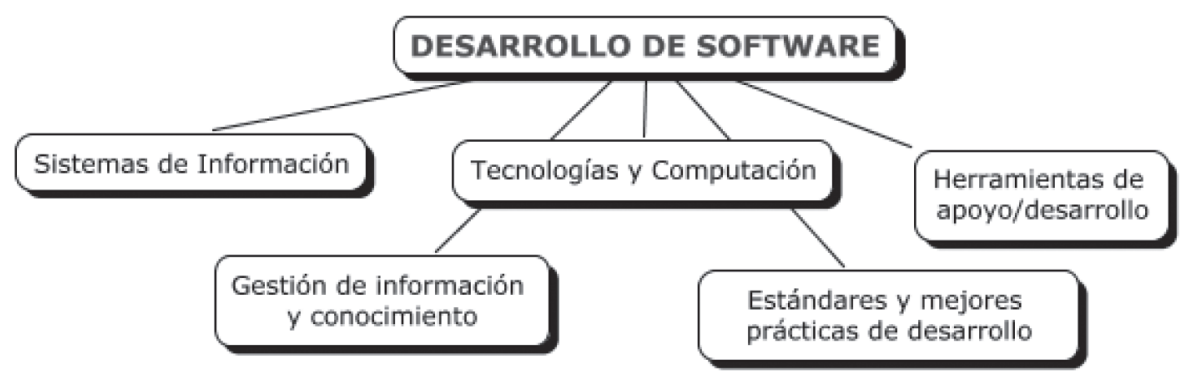

Figura 7. Mapa de la temática "Desarrollo de software".

socioeconómica, ubicación y nivel económico [11]. Para lo anterior es fundamental construir una plataforma educativa en la cual los conocimientos tengan significación, relevancia, capacidad de multiplicarse. Se plantean sub-temáticas como innovación educativa mediante la incorporación de las TIC, aspectos didácticos y pedagógicos para el uso y apropiación eficiente de estas tecnologías y diseño de portales educativos (Figura 9).

\section{- TIC y Medio ambiente}

Cualquier solución que involucre TIC debe cuidar aspectos medio-ambientales. Partiendo de esta base es fundamental investigar las mejores formas 


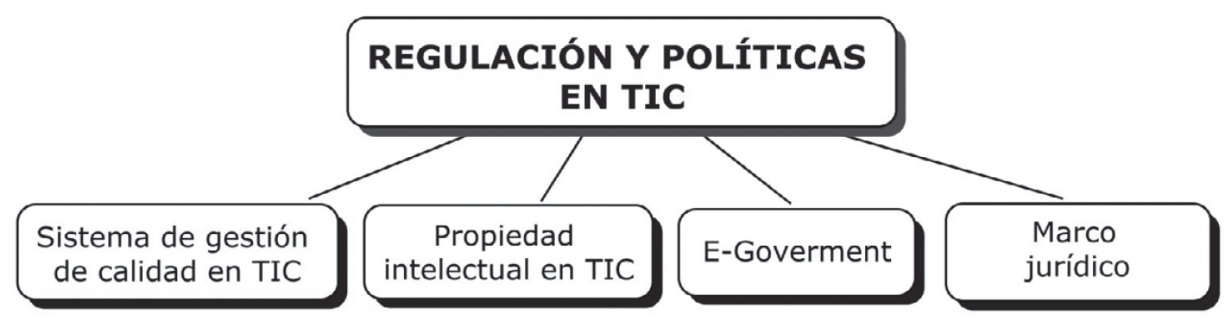

Figura 8. Mapa de la temática "Regulación y políticas en TIC".

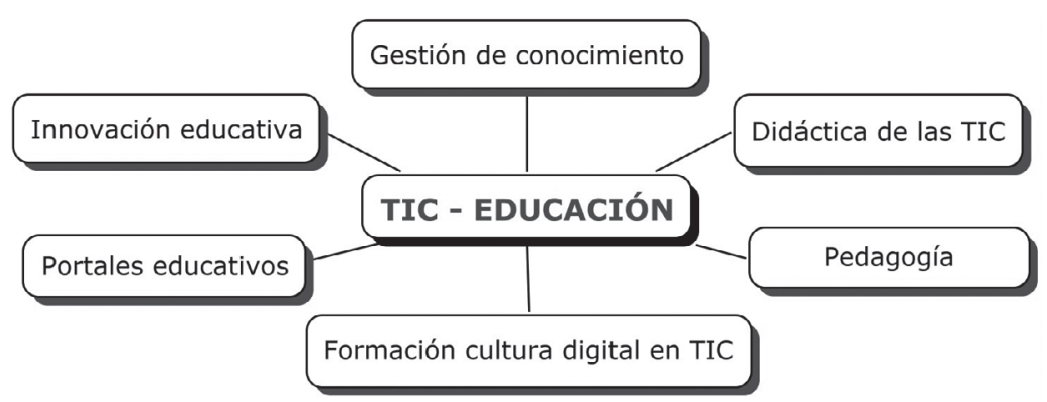

Figura 9. Mapa de la temática "TIC- Educación".

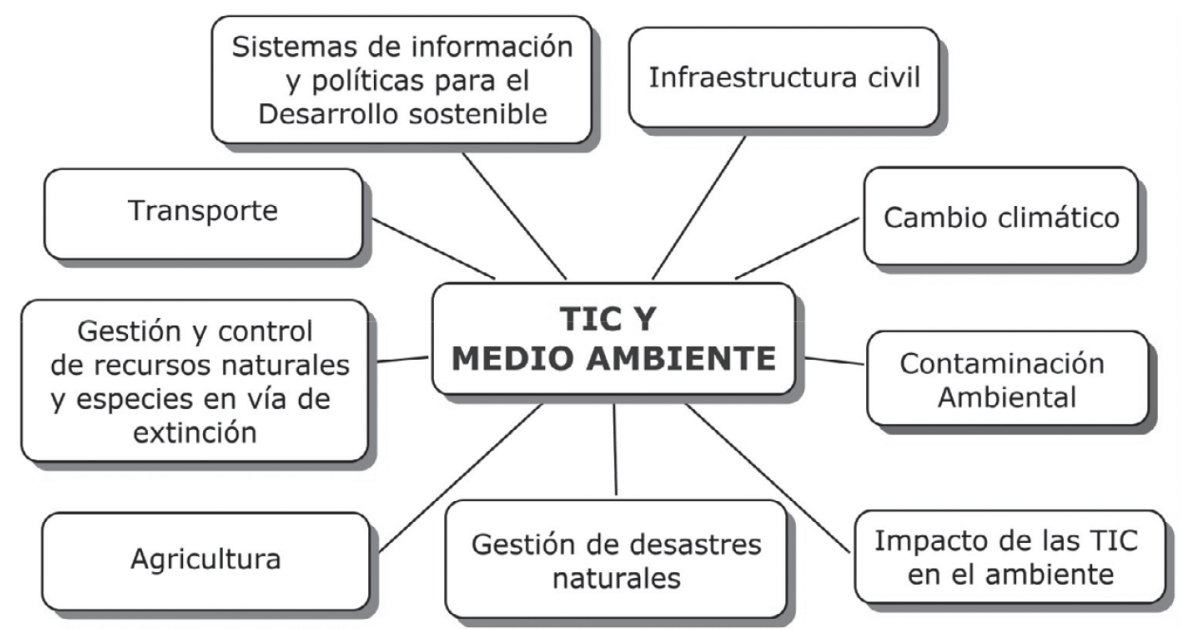

Figura 10. Mapa de la temática "TIC y medio ambiente".

de garantizar eficiencia energética, uso óptimo de recursos, la gestión de fuentes de poder adaptativas, entre otros. De igual manera se plantea como punto importante el análisis del impacto que tienen las TIC en la dinámica de procesos sociales y económicos de la región, buscando siempre que sean un elemento dinamizador. Es preciso disminuir el efecto que estas tecnologías pueden ocasionar sobre fenómenos como el cambio climático, la contaminación, buscando como filosofía que las nuevas soluciones en TIC aporten al desarrollo sostenible (Figura 10).

\section{- Contenidos digitales}

Este es un sector cada vez más estratégico para el desarrollo de los países y sus economías, ya que es fuente de riqueza para sus ciudadanos y mecanismo de apropiación de las TIC, así como un medio de promoción de su patrimonio cultural. 


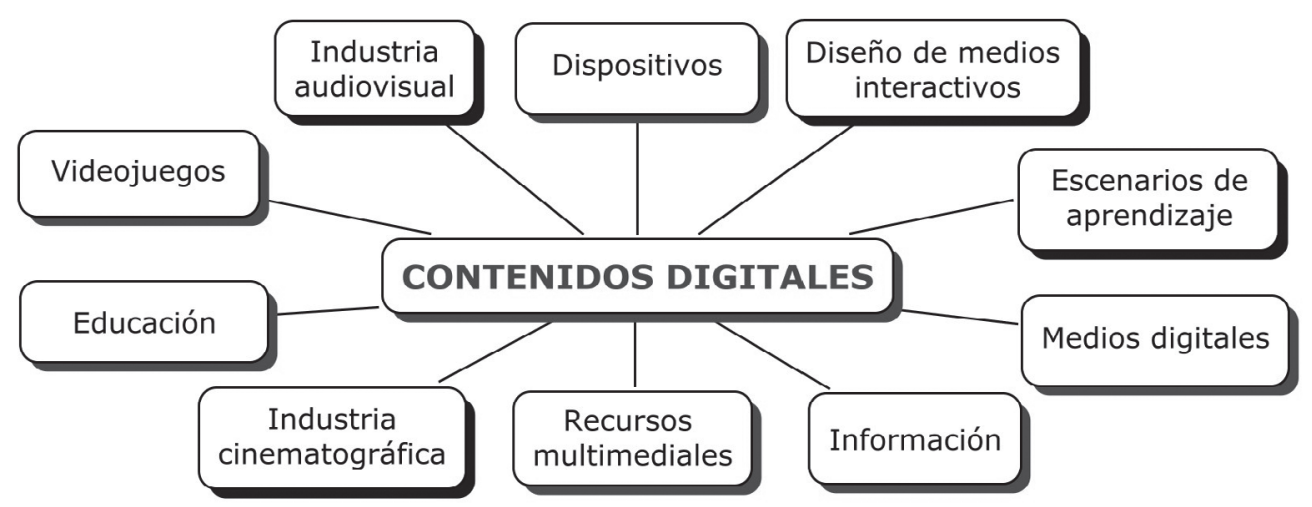

Figura 11. Mapa de la temática "Contenidos digitales"

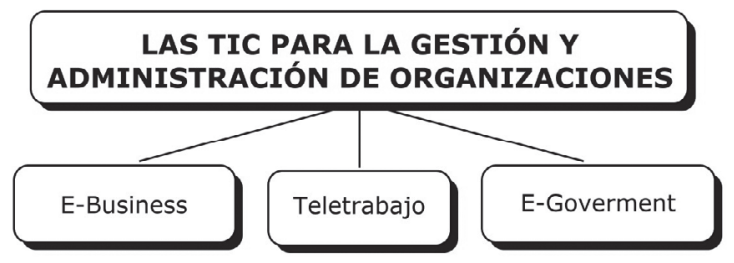

Figura 12. Mapa de la temática "Las TIC para la gestión y administración de la organizaciones".

Se trata de un segmento de las TIC con alto componente creativo, dinámico y cambiante, donde las características de hace un tiempo ya no son válidas en el entorno digital [16]. Para la definición de los ejes estratégicos de investigación en esta temática se analizó la cadena de valor de la industria de contenidos digitales, encontrando temáticas para el fortalecimiento de los principales eslabones (Figura 11).

\section{Las TIC para la gestión y administración de las organizaciones}

Con el objeto de mejorar la forma de operación de las empresas de diversos sectores productivos, es necesario hallar innovaciones en modelos de negocio con las TIC incorporadas desde la base. Esto incluye temas tan importantes como la cooperación entre diversas organizaciones nacionales e internacionales, lo cual se logra mediante el uso de mecanismos ofrecidos por las nuevas tecnologías que permiten hacer frente a los actuales retos de competitividad y desarrollo sostenible [1]. Otro tópico por explorar es el relacionado con el tele-trabajo con todas las oportunidades que conlleva tener personal calificado sin preocuparse por su ubicación geográfica. En esta categoría también se encuentra el tema de e-gobierno (Figura 12).

\section{CONCLUSIONES}

En los últimos años el mundo ha sido testigo del rápido crecimiento de la Tecnología de la Información y las Comunicaciones cuya importancia es cada vez mayor por convertirse en un activo dinámico, estratégico e indispensable para el desempeño de la misión y el logro de los objetivos de todas las organizaciones.

Una gobernanza eficaz de las TIC en la Universidad de Medellín propicia su aprovechamiento completo pasando a desempeñar una función esencial en los procesos misionales de docencia, investigación, extensión e internacionalización.

En este artículo se presentó una estrategia adoptada en la Universidad para favorecer la gobernabilidad en TIC desde el enfoque de investigación, a través de la estructuración de un campo en Tecnologías de la Información y la Comunicación.

Como grandes tendencias de investigación en TIC se identificaron: infraestructura tecnológica, educación, medio-ambiente, desarrollo de software, regulación, contenidos digitales y administración de organizaciones. 
Sólo las organizaciones que sepan aprovechar las ventajas que ofrecen las TIC y la sociedad en red, y consigan reinventar sus productos y servicios, junto a sus modelos de negocio, lograrán sobrevivir en un entorno marcado por una enorme disponibilidad de ofertas y de canales innovadores centrados en el usuario, en un contexto globalizado donde lo digital traspasa fronteras, físicas y mentales, con tendencia a la ubicuidad y la convergencia.

Contar con líneas de investigación en TIC claramente establecidas en la Universidad de Medellín mejora su competitividad, facilita el acceso a nuevos mercados, el aprovechamiento eficiente de recursos tecnológicos y complementarios, y como consecuencia, se incrementa el nivel de innovación y desarrollo tecnológico del sector y de Antioquia. El producto de esta investigación se constituye en una estrategia para favorecer la gobernanza de las TIC en la Institución, enfocando la capacidad instalada en término de recurso humano calificado, infraestructura y procesos; buscando construir un nicho en el que la Universidad de Medellín sea reconocida y claramente diferenciada con respecto a otras instituciones de educación superior.

Como trabajo futuro es necesario validar las tendencias identificadas con públicos externos, construir un mapa de campo que permita tener una visión de los actores que aportan a cada tendencia al interior de la organización y desarrollar un plan de comunicación para la difusión y apropiación del campo TIC en todos los niveles.

\section{AGRADECIMIENTOS}

Reconocimiento especial a la Universidad de Medellín a través de la Vicerrectoría de Investigación por la financiación de este proyecto, y a los grupos de investigación por validar parcialmente los resultados obtenidos.

\section{REFERENCIAS}

[1] Senado República de Colombia. "Ley $\mathrm{N}^{\circ}$ 1341. Principios y conceptos sobre la sociedad de la información y la organización de las Tecnologías de la Información y las Comunicaciones -TIC-". 2009.

[2] P. Weill and J. Ross. "IT Governance: How Top Performers Manage IT for Superior
Results". Harvard Business School Press. Vol. 1. Boston, Massachusetts, Estados Unidos, pp. 277. 2004. ISBN: 1-59139-253-5.

[3] Australian Computer Society. "The ICT Profession Body of Knowledge". Australian Computer Society. Sydney, Australia. 2012.

[4] E. Schiavo. "Investigación científica y tecnológica en el campo de las TIC: ¿conocimientos técnicos, contextuales o transversales?". Revista iberoamericana de ciencia tecnología y sociedad. Vol. 9, pp. 91-113. Agosto 2007. ISSN: 1850-0013.

[5] Corporate governance of information technology. International Organization for Standarization ISO ISO/IEC 38500-2008, 2008.

[6] Proyecto Educativo Institucional, Consiliatura Universidad de Medellín Decreto 3-Acta 615. Medellín, Colombia. 2010.

[7] L. Johnson, R. Smith, H. Willis, A. Levine and K. Haywook. "The 2011 Horizon Report". The New Media Consortium, EDUCAUSE Learning Initiative. Texas, Estados Unidos. 2011.

[8] Vicerrectoría de Investigaciones UdeM. "Consejería científica". Universidad de Medellín. Medellín, Colombia. 2008.

[9] H. Gadamer. "Verdad y método II". Ediciones Sígueme Salamanca. Edición 1, pp. 430. Salamanca, España. 1992. ISBN: 84-301-1180-8.

[10] E. González. "Sobre la hermenéutica o acerca de las múltiples lecturas de lo real". Sello Editorial Universidad de Medellín. Edición 1, pp. 120. Medellín, Colombia. 2006. ISBN: 958-97766-0-4.

[11] Secretaría de Educación de Antioquia. "Antioquia Virtual Siglo XXI". Julio 2011. Fecha de consulta: Mayo 2012. URL: http:// www.seduca.gov.co/index.php/antioquiavirtual-siglo-xxi.html

[12] Alcaldía de Medellín. "Medellín digital". Alcaldía de Medellín, pp. 19. Antioquia, Colombia. 2008.

[13] Ministerio de Tecnologías de la Información y las Comunicaciones. "Vive Digital Colombia". MinTIC. Bogotá, Colombia. 2011.

[14] L. Pineda and C. Scheel. "Plan estratégico de ciencia, tecnología e innovación de Medellín 2011-2021”. Ruta N. Medellín, Colombia. 2010. 
[15] W. Ríos. "La propiedad intelectual en la era de las tecnologías". Ediciones Uniandes. Vol. 1, pp 676. Bogotá, Colombia. 2011. ISBN: 978-958-35-0704-5.
[16] Clúster Audiovisual de Madrid. "Estudio sobre el estado actual y perspectivas futuras de los contenidos digitales". Red de parques y Clústers de la comunidad de Madrid. España. 2009. 